\title{
Filamentous phages of Ralstonia solanacearum: double-edged swords for pathogenic bacteria
}

\author{
Takashi Yamada* \\ Department of Molecular Biotechnology, Graduate School of Advanced Sciences of Matter, Hiroshima University, Higashi-Hiroshima, Japan
}

\section{Edited by:}

Heather K. Allen, National Animal Disease Center, USA

\section{Reviewed by:}

Loren John Hauser, The University of Tennessee, Oak Ridge National Laboratory, USA

Jasna Rakonjac, Massey University, New Zealand

\section{*Correspondence:}

Takashi Yamada, Department of Molecular Biotechnology, Graduate School of Advanced Sciences of

Matter, Hiroshima University, 1-3-1 Kagamiyama, Higashi-Hiroshima

739-8530, Japan

e-mail: tayamad@hiroshima-u.ac.jp
Some phages from genus Inovirus use host or bacteriophage-encoded site-specific integrases or recombinases establish a prophage state. During integration or excision, a superinfective form can be produced. The three states (free, prophage, and superinfective) of such phages exert different effects on host bacterial phenotypes. In Ralstonia solanacearum, the causative agent of bacterial wilt disease of crops, the bacterial virulence can be positively or negatively affected by filamentous phages, depending on their state. The presence or absence of a repressor gene in the phage genome may be responsible for the host phenotypic differences (virulent or avirulent) caused by phage infection. This strategy of virulence control may be widespread among filamentous phages that infect pathogenic bacteria of plants.

\section{Keywords: filamentous phage, integration, phytopathogen, Ralstonia solanacearum, virulence change}

\section{FILAMENTOUS PHAGES AND PATHOGENIC BACTERIA}

Bacteriophages belonging to the genus Inovirus are filamentous particles containing a circular single-stranded (ss) DNA genome. Infection with this kind of phage does not cause host cell lysis, but establishes a persistent association between the host and phage, producing and releasing phage particles from the growing and dividing host cells. In general, the genome of inoviruses, represented by Escherichia coli F-pillus specific phage Ff (f1, fd or M13), is organized in a modular structure, in which functionally related genes are grouped together (Horiuchi et al., 2009; Rakonjac et al., 2011). Three functional modules are always present: the replication module, the structural module, and the assembly and secretion module. The replication module contains the genes encoding rolling-circle DNA replication and singlestrand DNA (ssDNA) binding proteins $g I I, g V$, and $g X$ (Horiuchi et al., 2009). The structural module contains genes for the major $(g V I I I)$ and minor coat proteins ( $g I I I, g V I, g V I I$, and $g I X)$, and gene gIII encodes the host recognition or adsorption protein pIII (Wang et al., 2006). The assembly and secretion module contains the genes for morphogenesis and extrusion of the phage particles ( $g I$ and $g I V$; Marvin, 1998). Gene $g I V$ encodes protein pIV, an aqueous channel (secretin) in the outer membrane, through which phage particles exit from the host cells (Marciano et al., 1999). Some phages encode their own secretins, whereas others use host products (Davis et al., 2000).

Because inoviruses coexist with their host cells, infection by these phages can mediate conversion of the host bacterial phenotypes in various ways. In pathogenic bacteria of either animals or plants, virulence is frequently affected by phage infection. For example, infection of Xanthomonas campestris pv. oryzae NP5850 by the filamentous phages Xf and Xf2 enhanced virulence, possibly because of overproduction of extracellular polysaccharides (EPS) by the phage-infected bacterial cells (Kamiunten and Wakimoto, 1982). Tseng et al. (1990) also reported that infection of $X$. campestris pv. campestris by the filamentous phage Lf increased virulence by promoting EPS production. Filamentous phages are assembled at the host cell surface and secreted into the environment. However, once then cells form colonies on the semi-solid medium (and possibly within the liquid medium), some fractions of secreted phage population are bound to stay trapped in the colony, potentially accumulating to high concentrations and forming a matrix surrounding the cells in the colony. These trapped phage particles may serve to cross-link cells to give high densities and induce biofilms. This situation was reported for small colony variant formation in Pseudomonas aeruginosa depending on phage Pf4 activity (Webb et al., 2004; Rice et al., 2009). More direct involvement of filamentous phages in host virulence is well characterized in Vibrio cholerae. The pathogenicity of this severe diarrheal disease-causing bacterium depends on two key virulence factors, the toxin co-regulated pilus (TCP) and cholera toxin. Cholera toxin genes are encoded on the filamentous phage CTX $\phi$ and introduced into bacterial cells by phage integration mediated by the host dif/XerCD recombinase system (Huber and Waldor, 2002; Davis and Waldor, 2003). In Ralstonia solanacearum, infection by $\phi$ RSS1 induced the early expression of $p h c A$, a global virulence regulator, and also enhanced twitching motility (Addy et al., 2012b).

Contrasting with these virulence-enhancing effects of $\phi R S S 1$, loss of virulence was also reported in $R$. solanacearum. $R$. solanacearum completely lost virulence through infection with two other filamentous phages $\phi$ RSM1 and $\phi$ RSM3 (Addy et al., 2012a). Many virulence factors were significantly reduced in 
$\phi$ RSM-infected cells. These opposing effects of different filamentous phages on $R$. solanacearum virulence makes it an ideal study model system for understanding the effect of filamentous phage on their hosts. Here I will describe the role of filamentous phage in the virulence of $R$. solanacearum and suggest a causative relationship between a phage-encoded transcriptional repressor and $R$. solanacearum pathogenicity.

\section{Ralstonia solanacearum AND BACTERIAL WILT}

Ralstonia solanacearum is a Gram-negative $\beta$-proteobacterium that causes bacterial wilt disease in many important crops including tomato, potato, tobacco, and eggplant. Because of its wide geographic distribution and unusually broad host range (more than 50 plant families), it is responsible for significant crop losses worldwide (Hayward, 2000). Once the bacteria enter a susceptible host, they colonize the intercellular spaces of the root cortex and vascular parenchyma. The bacteria eventually enter the xylem and spread into the upper parts of the plant, causing wilt (Vasse et al., 2000; Kang et al., 2002; Yao and Allen, 2007). The development of bacterial wilt disease depends on bacterial pathogenicity and virulence (Carney and Denny, 1990; Denny, 2006). R. solanacearum virulence is additive, complex, and involves the production of multiple virulence factors (Schell, 2000; Genin and Boucher, 2002). For example, exopolysaccharide I (EPSI), a large nitrogen-rich acidic exopolysaccharide (Lavie et al., 2002), is thought to be an important virulence factor. It enhances the speed and extent of stem infection spreading from the root (Saile et al., 1997) and is presumed to cause wilting by restricting water flow through xylem vessels (Garg et al., 2000). In addition to EPSI, R. solanacearum secretes enzymes that degrade the plant cell wall through the type II secretion system (T2SS). Pectinolytic enzymes fragment pectin into oligomers, which act as a substrate for bacterial growth (TansKersten et al., 2001). The breakdown of pectin enhances virulence by facilitating bacterial movement through pectin-rich regions such as vascular bundles (Gonzalez and Allen, 2003). Cellulolytic enzymes also facilitate bacterial invasion of roots and/or penetration of xylem vessels by degrading cellulosic glucans in the cell wall (Liu et al., 2005). In addition to T2SS-mediated secreted proteins, the type IV pilus (Tfp) is believed to be another virulence factor of $R$. solanacearum (Davis and Waldor, 2003). This protein forms a surface appendage that is responsible for twitching motility and polar attachment to host cells or to plant roots, and enhances the severity of wilt disease (Liu et al., 2001; Kang et al., 2002).

Expression of the pathogenesis and virulence genes in $R$. solanacearum is controlled by a complex regulatory network (Schell, 2000; Genin and Boucher, 2002; Denny, 2006) and is drastically affected by various environmental factors. The regulation is outlined as follows: the transcriptional regulator PhcA plays a critical role in the regulatory network. Abundant PhcA activates production of multiple virulence factors such as EPSI and cell wall degrading enzymes (CWDE). PhcA is activated by a quorum sensing system mediated by the two-component regulatory system PhcS/PhcR that responds to thereshold levels of 3-OH palmitic acid methylester (3-OH PAME), an autoinducer of quorum sensing that controls virulence. Therefore, the levels of $3-\mathrm{OH}$ PAME, cell density, as well as cell surface nature all affect virulence in R. solanacearum.

\section{THREE STATES OF FILAMENTOUS PHAGE $\phi R S S$ WITH DIFFERENT EFFECTS ON HOST VIRULENCE}

$\phi R S S 1$ was isolated from a soil sample collected from tomato crop fields (Yamada et al., 2007). $\phi R S S 1$ particles have a flexible filamentous shape $1,100 \pm 100 \mathrm{~nm}$ in length and $10 \pm 0.5 \mathrm{~nm}$ in width, giving a morphology resembling coliphage $\mathrm{Ff}$ (M13, f1 or fd; Buchen-Osmond, 2003; ICTVdB). The $\phi R S S 1$ particles contain a ssDNA genome (6,662 nt; DDBJ accession no. AB259124), with a GC content of $62.6 \%$. There are 11 open reading frames (ORFs), located on the same strand (Figure 1A). The $\phi$ RSS1 gene arrangement is consistent with the general arrangement of Ff phages. Genomic Southern blot hybridization showed several examples of $\phi R S S 1$-related sequences integrated in the genomes of various $R$. solanacearum strains (Yamada et al., 2007). A $\phi R S S 1$-related phage (designated $\phi \mathrm{RSS} 0$ ) was induced and isolated from one such crosshybridizing strain (C319) by infection with another phage (jumbo phage $\phi R S L 1)$. The DNA sequence of $\phi R S S 0$ was very similar to $\phi R S S 1$, but contained an extra 626 nt at $\phi$ RSS1 position 6,628 , next to the intergenic region (IG), giving an entire genomic size of 7,288 nt (GenBank accession no. JQ408219). Within the $\phi$ RSS0 extra region, an ORF (ORF13) of $468 \mathrm{nt}$, corresponding to 156 amino acid residues, in a reversed orientation compared with the other ORFs, was found (Figure 1A). The amino acid sequence of ORF13 showed similarity to DNA-binding phage transcriptional regulators (accession no. B5SCX5, E-value = 1e-29).

Using inverse PCR with the new phage nucleotide sequences as primers, the prophage ( $\phi \mathrm{RSS} 0$ )-junctions ( $a t t \mathrm{~L}$ and $a t t \mathrm{R})$ in strain C319 were obtained and their nucleotide sequences determined. It was found that both $a t t \mathrm{~L}$ and $a t t \mathrm{R}$ contained repeated elements, corresponding to the dif sequence of $R$. solanacearum GMI1000 (Carnoy and Roten, 2009). This repeated sequence, 5'TATTT AACAT AAGAT AAAT- $3^{\prime}$, was also found at the $3^{\prime}$ end of ORF13 on the RSS0 genome, suggesting that it serves as attP.

Taken together, these results indicated that $\phi$ RSS1 (with a genome size of $6,662 \mathrm{nt}$ ) is a truncated form of the larger phage $\phi$ RSS0 (with a genome size of 7,288 nt). The 626 nt $\phi$ RSS0 sequence missing from $\phi \mathrm{RSS} 1$ contains attP (corresponding to the dif sequence) and ORF13, a possible regulatory gene (Yamada, 2011; Tasaka et al., unpublished). $\phi R S S 0$ is integrated at the dif site, similar to CTX $\phi$ of $V$. cholerae, which uses the host XerCD recombination system (Huber and Waldor, 2002). ORF13 encoded on $\phi$ RSS0 may function as a phage immunity factor, because strain C319 ( $\phi$ RSS0 lysogen) is resistant to secondary infection by $\phi \mathrm{RSS} 0$. C319 is susceptible to $\phi \mathrm{RSS} 1$, thus $\phi \mathrm{RSS} 1$ (without ORF13) may be an escaped superinfective phage. These three states of $\phi R S S$ phages and their interrelationships are shown in Figure 1B.

Upon infection by the $\phi$ RSS1 phage, the host $R$. solanacearum cells showed several abnormal behaviors, including less turbidity and frequent aggregation in the liquid culture, less coloration of colonies on plates, and a decreased growth rate (approximately $60 \%$ of the normal rate). More interestingly, $\phi$ RSS1-infected cells showed enhanced virulence on tobacco (Yamada et al., 2007) and tomato plants (Addy etal., 2012b). In the case of strain C319 ( $\phi$ RSS0 lysogenic), inoculated tobacco plants showed wilting symptoms of grade 2-3 at 14 days post-inoculation (p.i.), whereas tobacco plants inoculated with $\phi$ RSS1-infected C319 cells 

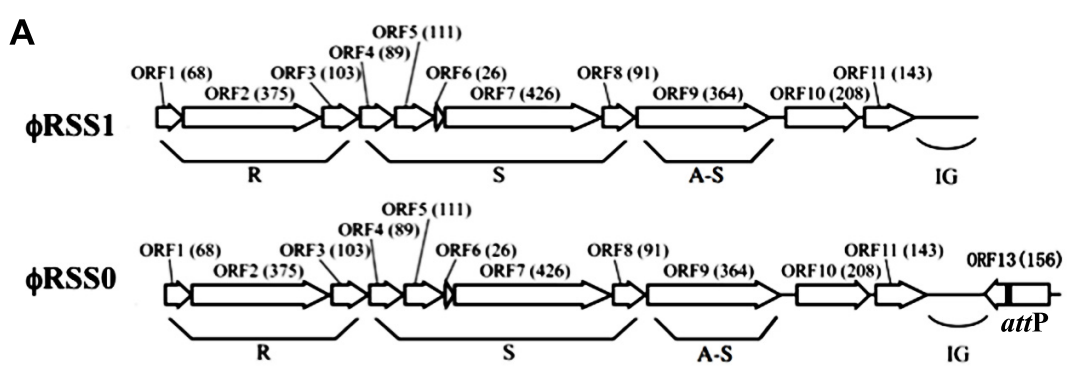

B

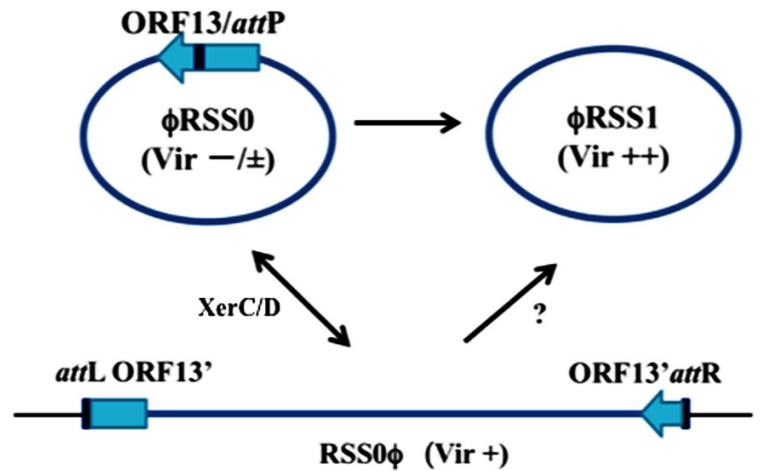

FIGURE $1 \mid \phi R S$ type filamentous phages infecting $R$. solanacearum. (A) Genomic organization of $\phi$ RSS1 and $\phi$ RSSO (Kawasaki etal., 2007; Yamada, 2011) shown in a linear form. ORFs or genes are represented by arrows oriented in the direction of transcription. The functional modules for replication $(R)$, structure $(S)$, and assembly secretion $(A-S)$ are indicated according to the M13 model (Marvin, 1998). The region containing the attP sequence is also indicated. (B) Interrelationship between three states of $\phi R S S$ phages. The phage genomic DNA is shown in a circular form where most genes are not shown. $\phi R S S 0$ is equipped with a 626-nt element containing ORF13, within which attP (dif) is located. This element is missing in $\phi R S S 1$. The processes of interconversion between $\phi R S S 0$ and $\phi R S S 1$ are not known. $\phi R S S 0$ is integrated at the dif site (attB) on the host genome. The prophage state is shown as RSSO $\phi$, where the left and right borders are indicated as att $L$ and att $R$, respectively. This integration (reversible) is mediated by the host XerCD system. $\phi$ RSS1 may be produced directly from RSSO $\phi$. The three states of $\phi R S S 0$-type phage ( $\phi \mathrm{RSSO}, \phi \mathrm{RSS} 1$, and $\phi \mathrm{RSSO}$ prophage) affect host $R$. solanacearum cells differently after infection, especially in host virulence. Compared with wild-type virulence $(+)$, $\phi$ RSS1 enhances $(++)$ and $\phi$ RSSO reduces $(-/ \pm)$ the host virulence. wilted earlier; grade 2-3 symptoms were observed at 10 days p.i. and plants were almost dead at 14 days p.i. (Yamada et al., 2007). Effects on host virulence by infection with $\phi$ RSS0 in its free form (not prophage) were also examined. To make wilting symptoms clear, tomato-tropic R. solanacearum strain (MAFF 106603) in tomato experimental system was used. The cells were infected with either $\phi R S S 0$ (free) or $\phi$ RSS1. The physiological features of $\phi R S S 0$-infected $R$. solanacearum MAFF 106603 cells were almost the same as $\phi R S S 1$-infected MAFF 106603 cells, except that the $\phi R S S 0$-infected cells formed colonies of more mucoid appearance on CPG plates. When MAFF 106603 (wild-type) cells were inoculated into the major stem of tomato plants, all 12 plants showed wilting symptoms as early as 3 days p.i. and died 5-7 days p.i. $\phi R S S 1$-infected cells of MAFF 106603 inoculated into tomato in the same way caused wilting earlier, at 2 days p.i., and all 12 plants died by 5 days p.i. In contrast, tomato plants inoculated with $\phi R S S 0$-infected cells showed wilting symptoms much later: most plants (10 of 12) survived after 7 days and a few plants did not show any symptoms until 23 days p.i. Therefore, $\phi$ RSS0 infection caused reduced virulence in host bacterial cells (Tasaka et al., unpublished). The virulence-enhancing effects by $\phi$ RSS 1 infection can be explained as follows: surface-associated $\phi$ RSS1 particles (or phage proteins) may change the surface nature (hydrophobicity) of host cells to generate a high local cell density, resulting in early activation of $p h c A$, the global virulence regulator, or lack of orf13, which is absent from the $\phi$ RSS1 genome (Addy et al., 2012b). The reduced virulence observed for $\phi$ RSS0-infected cells may be caused by the function(s) of ORF13 encoded by $\phi$ RSS 0 . These results are summarized in Table $\mathbf{1}$.

\section{EFFECT OF FILAMENTOUS PHAGE $\phi R S M$ ON VIRULENCE OF Ralstonia solanacearum}

$\phi R S M 1$ is also a soil-isolated filamentous phage $1,400 \pm 300 \mu \mathrm{m}$ long and $10 \pm 0.7 \mathrm{~nm}$ wide (Yamada et al., 2007). The infection cycle of $\phi R S M 1$ phage resembles that of $\phi R S S 1$. The genome of фRSM1 is 9,004 nt long (DDBJ accession No. AB259123) with

Table 1 | Three states of filametous phages and their effects on host virulence.

\begin{tabular}{|c|c|c|c|}
\hline Phage state & $\phi$ RSS-type & $\phi R S M-t y p e$ & Virulence \\
\hline Free & $\phi$ RSSO & $\phi \mathrm{RSM} 3$ & $+/-$ or - \\
\hline Prophage & $\mathrm{RSSO} \phi$ & RSM3 $\phi$ & + \\
\hline Superinfective mutant & $\phi$ RSS1 & $\phi$ RSM3- $\triangle$ ORF15 & ++ \\
\hline
\end{tabular}


a GC content of $59.9 \%$. There are 12 putative ORFs located on the same strand and three on the opposite strand. The $\phi \mathrm{RSM} 1$ genes are shown in Figure 2A, in comparison with the conserved gene arrangement of M13-like phages (Kawasaki etal., 2007). Here, ORF13, ORF14, and ORF15 (reversely oriented) are inserted between ORF12, corresponding to pII as a replication protein, and ORF1, corresponding to a ssDNA-binding protein like $\mathrm{pV}$, in the putative replication module. ORF13, ORF14, and ORF15 show amino acid sequence similarity to a proline-rich transmembrane protein, a resolvase/DNA invertaselike recombinase, and a putative phage repressor, respectively (Kawasaki et al., 2007; Addy et al., 2012a). There are two additional ORFs (ORF2 and ORF3) between the replication and structural modules. The functions of these ORF-encoded proteins are not known. In genomic Southern blot hybridization, two different types of $\phi R S M 1-$ related prophage sequences were detected in R. solanacearum strains. Strains of type A include MAFF211270 and produce $\phi R S M 1$ itself, and strains of type B (giving different restriction patterns) are resistant to $\phi \mathrm{RSM} 1$ infection, but are susceptible to $\phi$ RSM3 (see below). By determining the nucleotide sequences of junction regions of the $\phi R S M 1$-prophage in the MAFF 211270 chromosomal DNA, an att $\mathrm{P} /$ att $\mathrm{B}$ core sequence was identified as $5^{\prime}$-TGGCGGAGAGGGT-3', corresponding to positions $8,544-8,556$ of $\phi R S M 1$ DNA, located between ORF14 and ORF15. Its nucleotide sequence is identical to the $3^{\prime}$-end of the host $R$. solanacearum gene for serine tRNA(UCG) in the reverse orientation. A $\phi R S M 1-l i k e$ prophage (type B) in strain MAFF 730139, designated $\phi$ RSM3, was obtained by PCR amplification using appropriate primers containing these att sequences (Askora et al., 2009). Compared with the $\phi R S M 1$ genome, the $\phi R S M 3$ prophage sequence $(8,929 \mathrm{nt})$ is $75 \mathrm{nt}$ shorter. The sequences show 93\% nucleotide identity and major differences are found within two regions; positions 400-600 and positions $2,500-3,000$ in the $\phi R S M 1$ sequence. The former region corresponds to ORF2, which is inserted between the replication module $(\mathrm{R})$ and the structural module (S), and has no similarity between the two phages. The latter falls into the possible D2 domain of ORF9 (pIII), which determines the host range. All other ORFs identified along the $\phi$ RSM3 are highly conserved between two phages (over 90\% amino acid identity). It is interesting that the amino acid sequence of ORF14 (putative DNA invertase/recombinase) is $100 \%$ identical in the two phages. The gene arrangement of $\phi R S M 3$, which is almost the same as $\phi R S M 1$, is shown in Figure 2A.

As described above, the genomes of $\phi \mathrm{RSM}$ phages are sometimes integrated in the host genome. Askora et al. (2011) demonstrated that the integration is mediated by the phageencoded recombinase (ORF14 of $\phi R \mathrm{RSM} 1 / \phi \mathrm{RSM} 3$ ), which has significant homology to resolvases/DNA invertases (small serine recombinases), with att $\mathrm{P} /$ att $\mathrm{B}$ corresponding to the $3^{\prime}$ end of the host serine tRNA(UCG) gene in the reverse orientation. This is the first case of filamentous phages demonstrated to integrate into the host genome by its endogenously encoded integrase (Askora etal., 2012). The same unit of integration ( $\phi \mathrm{RSM}$ Int/attP) was found in a Ralstonia pickettii 12J phage and in Burkholderia pseudomallei 668 prophages (Askora et al., 2012). Together with these phages, it would not be surprising if similar Int-containing filamentous phages occur widely in nature.

Infection by $\phi \mathrm{RSM} 1$ or $\phi \mathrm{RSM} 3$ establishes a persistent association between the host and the phage. Upon infection by $\phi$ RSM phages, the host cells showed some abnormal behaviors and characteristics, such as frequent aggregation, dark coloration, and relatively small colony size, as observed in $\phi$ RSS infection. When cells of MAFF 106611 ( $\phi$ RSM3 lysogenic strain) or MAFF 106603 (not lysogenic) were inoculated into tomato plants, all 20 plants showed wilting symptoms as early as 3 days p.i., whereas none of the 20 tomato plants inoculated with free- $\phi R S M 3$-infected cells (for example, MAFF 106603) showed any wilting symptoms until 4 weeks p.i. (Addy et al., 2012a). This loss of virulence effect of $\phi R S M 3$ infection can be explained in three ways: (i) reduced twitching motility and reduced amounts of type IV pili (Tfp), (ii) lower levels of $\beta$-1,4-endoglucanase (Egl) activity and EPS production, and (iii) reduced expression of certain virulence/pathogenicity genes (egl, pehC, phcA, phcB, pilT, and $h r p B$ ) in the infected cells (Addy et al., 2012a). This is supported restoring virulence in $\phi R S M 3$ lysogen by deletion of $\phi R S M 3-$ encoded orf15, the gene for a putative repressor-like protein, was disrupted (Addy et al., 2012a). Therefore, ORF15 of $\phi$ RSM3 may repress host genes involved in pathogenicity/virulence and consequently result in loss of virulence. With different strains as hosts, $\phi R S M 1$ also gave similar results. The $\phi R S M$ states and interaction with the host genome can be depicted similarly to $\phi$ RSS phages, as shown in Figure 2B. These results are summarized and compared with the three states of $\phi R S S$ in Table 1.

\section{PERSPECTIVE AND HYPOTHESIS}

As seen here, for $R$. solanacearum, filamentous phages such as $\phi R S S$ and $\phi R S M$ are double-edged swords; sometimes they help bacteria to infect plants by enhancing bacterial virulence, and sometimes they interrupt bacterial infection of plants by repressing host genes involved in virulence. The contradictory effects of these phages may largely depend on the presence or absence of a phage-encoded regulatory protein. Two questions arise here: (i) How does the regulatory affect on the host genes; working alone, with other phage factors, or with host factors? (ii) How does such a regulatory gene become acquired by or lost from the phage genome? Concerning the first question, as shown in Figure 1B, attP is located within ORF13 on $\phi R S S 0$ DNA, and after integration at attB on the host genome, a truncation of ORF13 (at the C-terminus) occurs. By creating a new stop codon in the reading frame, the size of ORF13 reduced from 156 to 130 aa with a 26-aa truncation at the C-terminus (Tasaka et al., manuscript in preparation). A DNA-biding motif (Helix-Turn-Helix) is located in the $\mathrm{N}$-terminal moiety and the $\mathrm{C}$-terminal region may have some regulatory function (such as ligand-binding). This suggests a functional difference of the ORF13 protein before and after integration. One possibility is that the full length ORF13 (ORF15 in $\phi R S M$ phages) expressed from free phages may function to preferentially regulate host genes and the truncated (or modified) form expressed from the prophage may function to stabilize the prophage state and phage immunity, protecting against infections by related phages (Hypothesis 1). This hypothesis is compatible 


\section{A}

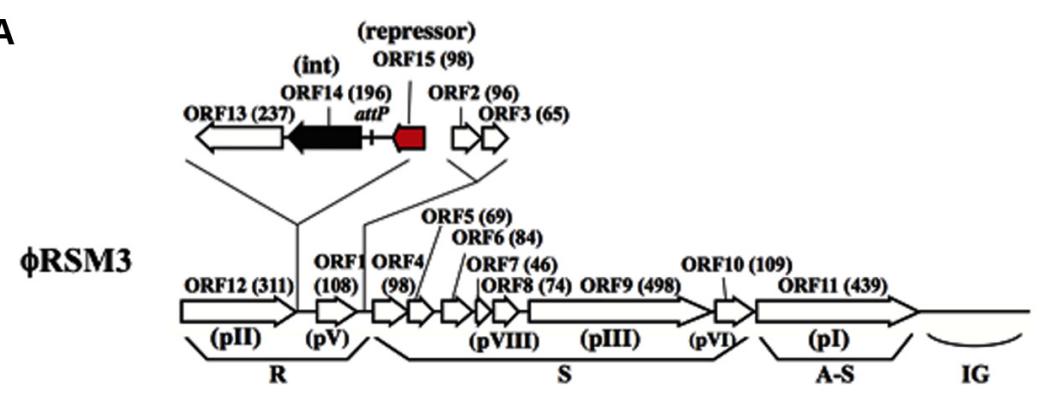

B

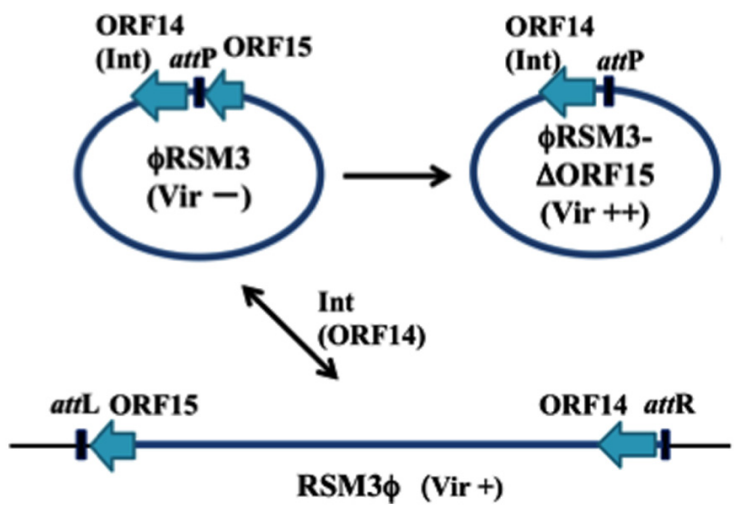

FIGURE 2 | $\phi R S M$ type filamentous phages infecting $R$. solanacearum. (A) Genomic organization of $\phi$ RSM1 (Kawasaki et al., 2007; Askora et al., 2011). Map is drawn in the same way as in Figure 1. ORF14 and ORF15 correspond to Int (resolvase/invertase-like serine recombinase) and a newly identified DNA-binding repressor-like protein (Addy et al., 2012a), respectively. attP corresponds to a 13-nt 3'-end of the serine tRNA(UCG). (B)

Interrelationship between three states of $\phi R S M$ phages. For the experimental convenience $\phi R S M 3$ was used in the study. The phage genomic DNA is shown in a circular form where most genes are not shown. $\phi R S M 3-\triangle \mathrm{ORF} 15$ is a deletion mutant of $\phi R S M 3$ lacking ORF15 (Addy etal., 2012a). $\phi R S M 3$ is integrated at the tRNA(UCG) site (attB) on the host genome. The prophage state is shown as RSM3 $\phi$, where the left and right borders are indicated as att $L$ and att $R$, respectively. This integration (reversible) is mediated by phage-encoded Int (ORF14). The three states of $\phi R S M$-type phage ( $\phi R S M 3$, $\phi R S M 3-\triangle O R F 15$, and RSM3 $\phi$ prophage) affect host $R$. solanacearum cells differently after infection, especially in host virulence. Compared with wild-type virulence $(+), \phi R S M 3-\Delta$ ORF15 enhances $(++)$ and $\phi R S M 3$ suppress (-) the host virulence. with the observation that once a $\phi \mathrm{RSS}$ and $\phi \mathrm{RSM}$ prophage state was established, the phage genomic DNA and phage particles seldom appeared in the lysogenic strains. Like $\phi R S M$, the DNA or the phage particles are not identified in the lysogen, even though the orf15 encoding the putative repressor ORF15 is not changed before and after the host integration. Because ORF14 integrase (serine recombinase) of $\phi \mathrm{RSM}$ phages likely mediates both integrative and excessive recombinations (Askora et al., 2011), some additional factors are required to mediate prophage replication or excision. The function, regulatory mechanism, and effect on virulence of $\phi R S S$ orf13 or $\phi$ RSM orf 15 remain to be investigated by direct expression of the corresponding gene in an appropriate host strain. In our preliminary trial where the coding region of ORF15 of $\phi$ RSM3 (ORF13 of $\phi$ RSS0) was expressed from a plasmid under the control of lacP and introduced into appropriate host strains, no transformants with a correct construct appeared (colonies that appeared on the selection plates after transformation all contained deleted inserts). One of the explanation for this is putative toxic effect of ORF13 and ORF15 on the host when expressed under these conditions. Some additional factors encoded on the phage genome may be involved in the appropriate regulation, interacting with ORF13 or ORF15 (Hypothesis 2). Further studies with mutated constructs of ORF13 or ORF15 are required to test these hypotheses.

As for question of loss of a repressor protein, a 626-nt sequence unit containing orf13 and attP detected in $\phi \mathrm{RSS} 0$ and missing from $\phi R S S 1$ plays a crucial role in $\phi R S S$ dynamics. The origin of such a sequence and the mechanism how it comes in or out of the phage are largely unknown. However, the possibility of two forms from a phage is important. Apparently, $\phi \mathrm{RSS} 1$-infected bacterial cells have an advantage in the pathogenic lifestyle. Nevertheless, the virulence is not always necessary for this soil-borne bacterium. Infection of $\phi R S S 0$ provides the host cells with a sophisticated mechanism to control their virulence. Similar mechanisms may function in other pathogenic bacteria (Hypothesis 3). To test this hypothesis, various systems involving pathogenic bacteria and their filamentous phages should be examined. For example, $\phi R S S 1-l i k e$ superinfective phage Cf1tv spontaneously appeared from the Cf1t lysogenic strain of Xanthomonas campestris pv. citri (Kuo et al., 1994). Unfortunately, nucleotide sequence information is not available for this phage. Similar kinds of phage involvement in host virulence regulation may be universal, because $\phi$ RSS- or $\phi R S M-r e l a t e d ~ s e q u e n c e s$ are frequently found in various bacterial genomic sequences in the databases, including $R$. pickettii 
(CP001645), Ralstonia syzygii (FR854090), Burkholderia rhizoxinica (FR687359), Pectobacterium wasabiae (CP001790), and Erwinia carotovora (BX950851). There are also other filamentous phages that have lysogenic cycles, including $X$. campestris phages Cf1c (Kuo et al., 1991), Cf1t (Kuo et al., 1987a,b), Cf16v1 (Dai et al., 1980), and $\phi$ Lf (Lin et al., 2000); Xylella fastidiosa phage Xf $\phi$-f1 (Simpson et al., 2000); Yersinia pestis phages CUS $\phi-2$ (Gonzalez and Allen, 2003) and Ypf $\phi$ (Derbise et al., 2007); Nf of Neisseria meningitidis (Kawai et al., 2005), and V. cholerae phages VGJ $\phi$ (Campos etal., 2003) and VCY $\phi$ (Xue etal., 2012). The host bacteria of these phages are plant or animal pathogens.

\section{ACKNOWLEDGMENTS}

This study was supported in part by Research and Development Projects for Application in Promoting New Policy of Agriculture, Forestry, and Fisheries (No. 250037B), and by JST/BIOTEC Strategic Research Cooperative Program on Biotechnology.

\section{REFERENCES}

Addy, H. S., Askora, A., Kawasaki, T., Fujie, M., and Yamada, T. (2012a). Loss of virulence of the phytopathogen Ralstonia solanacearum through infection by фRSM filamentous phages. Phytopathology 102, 469-477. doi: 10.1094/PHYTO11-11-0319-R

Addy, H. S., Askora, A., Kawasaki, T., Fujie, M., and Yamada, T. (2012b). The filamentous phage $\phi R S S 1$ enhances virulence of phytopathogenic Ralstonia solanacearum on tomato. Phytopathology 102, 244-251. doi: 10.1094/PHYTO-10-11-0277

Askora, A., Abdel-Haliem, M. E. F., and Yamada, T. (2012). Site-specific recombination systems in filamentous phages. Mol. Genet. Genomics 287, 525-530. doi: 10.1007/s00438-012-0700-1

Askora, A., Kawasaki, T., Fujie, M., and Yamada, T. (2011). Resolvase-like serine recombinase mediates integration/excision in the bacteriophage $\phi$ RSM. J. Biosci. Bioeng. 111, 109-116. doi: 10.1016/j.jbiosc.2010.10.001

Askora, A., Kawasaki, T., Usami, S., Fujie, M., and Yamada, T. (2009). Host recognition and integration of filamentous phage $\phi \mathrm{RSM}$ in the phytopathogen, Ralstonia solanacearum. Virology 384, 69-76. doi: 10.1016/j.virol.2008.11.007

Buchen-Osmond, C. (2003). "Inoviridae.” in ICTVdB-The Universal Virus Database, version 3. ed. A. Z. Oracle (USA: ICTVdB Management, The Earth Institute, Biosphere 2 Center, Columbia University).

Carney, B. F., and Denny, T. P. (1990). A cloned avirulence gene from Pseudomonas solanacearum determines incompatibility on Nicotiana tabacum at the host species level. J. Bacteriol. 172, 4836-4843.

Campos, J., Martinez, E., Suzarte, E., Rodriguez, B. E., Marrero, K., Silva, Y., et al. (2003). A novel filamentous phage of Vibrio cholerae, integrates into the same chromosomal site as CTXф. J. Bacteriol. 185, 5685-5696. doi: 10.1128/JB.185.19.5685-5696.2003

Carnoy, C., and Roten, C.-A. (2009). The dif/Xer recombination systems in Proteobacteria. PLoS ONE 4:e6531. doi: 10.1371/journal.pone.0006531

Dai, H., Chiang, K. S., and Kuo, T. T. (1980). Characterization of a new filamentous phage Cf from Xanthomonas citri. J. Gen. Virol. 46, 277-289. doi: 10.1099/00221317-46-2-277

Davis, B. M., Lawson, E. H., Sandkvist, M., Sozhamannan, S., Ali, A., and Waldor, M. K. (2000). Convergence of the secretory pathways for cholera toxin and the filamentous phage, СТX $\phi$. Science 288, 333-335. doi: 10.1126/science.288.5464.333

Davis, B. M., and Waldor, M. K. (2003). Filamentous phages linked to virulence of Vibrio cholerae. Curr. Opin. Microbiol. 6, 35-42. doi: 10.1016/S13695274(02)00005-X

Denny, T. P. (2006). "Plant pathogenic Ralstonia species," in Plant-Associated Bacteria. ed. S. S. Gnanamanickam (Amsterdam, Netherlands: Springer), 573-644.

Derbise, A., Chenal-Francisque, V., Pouillot, F., Fayolle, C., Prevost, M. C., Medigue, C., et al. (2007). A horizontally acquired filamentous phage $c$ ontributes to the pathogenicity of the plague bacillus. Mol. Microbiol. 63, 1145-1157. doi: 10.1111/j.1365-2958.2006.05570.x

Garg, R. P., Huang, J., Yindeeyoungyeon, W., Denny, T. P., and Schell, M. A. (2000). Multicomponent transcriptional regulation at the complex promoter of the exopolysaccharide I biosynthetic operon of Ralstonia solanacearum. J. Bacteriol. 182, 6659-6666. doi: 10.1128/JB.182.23.6659-6666.2000

Genin, S., and Boucher, C. (2002). Ralstonia solanacearum: secrets of a major pathogen unveiled by analysis of its genome. Mol. Plant Pathol. 3, 111-118. doi: 10.1046/j.1364-3703.2002.00102.x

Gonzalez, E. T., and Allen, C. (2003). Characterization of a Ralstonia solanacearum operon required for polygalacturonate degradation and uptake of galacturonic acid. Mol. Plant Microbe Interact. 16, 536-544. doi: 10.1094/MPMI.2003.16.6. 536

Hayward, A. C. (2000). "Ralstonia solanacearum," in Encyclopedia of Microbiology, Vol. 4, ed. J. Lederberg (San Diego, CA: Academic Press), 32-42.

Horiuchi, K., Volvis, G. E., and Model, P. (2009). "The filamentous phage genome: genes, physical structure, and protein products," in Cold Spring Harbor Monograph Archive (Cold Spring Harbor, NY: Cold Spring Harbor Laboratory Press), 113-137.

Huber, K. E., and Waldor, M. K. (2002). Filamentous phage integration requires the host recombinases XerC and XerD. Nature 417, 656-659. doi: 10.1038 /nature00782

Kamiunten, H., and Wakimoto, S. (1982). Effect of the infection with filamentous phage Xf-2 on the properties of Xanthomonas campestris var oryzae. Ann. Phytopathol. Soc. Jpn. 47, 627-636. doi: 10.3186/jjphytopath.47.627

Kang, Y., Liu, H., Genin, S., Schell, M. A., and Denny, T. P. (2002). Ralstonia solanacearum requires type 4 pili to adhere to multiple surfaces and for natural transformation and virulence. Mol. Microbiol. 46, 427-437. doi: 10.1046/j.13652958.2002.03187.x

Kawai, M., Uchiyama, I., and Kobayashi, I. (2005). Genome comparison in silico in Neisseria suggests integration of filamentous bacteriophages by their own transposase. DNA Res. 12, 389-401. doi: 10.1093/dnares/dsi021

Kawasaki, T., Nagata, S., Fujiwara, A., Satsuma, H., Fujie, M., Usami, S., et al. (2007). Genomic characterization of the filamentous integrative bacteriophage $\phi$ RSS1 and $\phi R S M 1$, which infect Ralstonia solanacearum. J. Bacteriol. 189, 5792-5802. doi: 10.1128/JB.00540-07

Kuo, T. T., Chao, Y. S., Lin, Y. H., Lin, B. Y., Liu, L. F., and Feng, T. Y. (1987a). Integration of the DNA of filamentous bacteriophage Cf1t into the chromosomal DNA of its host. J. Virol. 61, 60-65.

Kuo, T. T., Lin, Y. H., Huang, C. M., Chang, S. F., Dai, D., and Feng, T. Y. (1987b). The lysogenic cycle of the filamentous phage Cf1t from Xanthomonas campestris pv. citri. Virology 156, 305-312. doi: 10.1016/0042-6822(87)90410-7

Kuo, T. T., Chiang, C. C., Chen, S. Y., Lin, J. H., and Kuo, J. L. (1994). A long lytic cycle in filamentous phage Cf1tv infecting Xanthomonas campestris pv. citri. Arch. Virol. 135, 253-264. doi: 10.1007/BF01310012

Kuo, T. T., Tan, M. S., Su, M. T., and Yang, M. K. (1991). Complete nucleotide sequence of filamentous phage Cf1c from Xanthomonas campestris pv. citri. Nucleic Acids Res. 19, 2498. doi: 10.1093/nar/19.9.2498

Lavie, M., Shillington, E., Eguiluz, C., Grimsley, N., and Boucher, C. (2002). PopP1, a new member of the YopJ/AvrRxv family of type III effector proteins, acts as a host-specificity factor and modulates aggressiveness of Ralstonia solanacearum. Mol. Plant Microbe Interact. 15, 1058-1068. doi: 10.1094/MPMI.2002.15.10. 1058

Lin, N. T., Chang, R. Y., Lee, S. J., and Tseng, Y. H. (2000). Plasmids carrying cloned fragments of RF DNA from the filamentous phage $\phi L F$ can be integrated into the host chromosome via site-specific integration and homologous recombination. Mol. Genet. Genom. 266, 425-435.

Liu, H., Kang, Y., Genin, S., Schell, M. A., and Denny, T. P. (2001). Twitching motility of Ralstonia solanacearum requires a type IV pilus system. Microbiology 147, 3215-3229.

Liu, H., Zhang, S., Schell, M. A., and Denny, T. P. (2005). Pyramiding unmarked deletions in Ralstonia solanacearum shows that secreted proteins in addition to plant cell-wall-degrading enzymes contribute to virulence. Mol. Plant Microbe Interact. 18, 1296-1305. doi: 10.1094/MPMI-18-1296

Marciano, D. K., Russel, M., and Simon, S. M. (1999). An aqueous channel for filamentous phage export. Science 284, 1516-1519. doi: 10.1126/science.284.5419.1516

Marvin, D. A. (1998). Filamentous phage structure, infection and assembly. Curr. Opin. Struct. Biol. 8, 150-158. doi: 10.1016/S0959-440X(98)80032-8

Rakonjac, J., Bennet, N. J., Spagnuolo, J., Gagic, D., and Russel, M. (2011). Filamentous bacteriophage: biology, phage display and nanotechnology applications. Curr. Issues Mol. Biol. 13, 51-76. doi: 10.1002/9780470015902.a0000777 
Rice, S. A., Tan, C. H., Mikkelsen, P. J., Kung, V., Woo, J., Tay, M., et al. (2009). The biofilm life cycle and virulence of Pseudomonas aeruginosa are dependent on a filamentous prophage. ISME J. 3, 271-282. doi: 10.1038/ismej.2008. 109

Saile, E., McGarvey, J. A., Schell, M. A., and Denny, T. P. (1997). Role of extracellular polysaccharide and endoglucanase in root invasion and colonization of tomato plants by Ralstonia solanacearum. Phytopathology 87, 1264-1271. doi: 10.1094/PHYTO.1997.87.12.1264

Schell, M. A. (2000). Control of virulence and pathogenicity genes of Ralstonia solanacearum by an elaborate sensory array. Annu. Rev. Phytopathol. 38, 263-292. doi: 10.1146/annurev.phyto.38.1.263

Simpson, A. J., Reinach, F. C., Arruda, P., Abreu, F. A., Acencio, M., Alvarenga, R., et al. (2000). The genome sequence of the plant pathogen Xylella fastidiosa. Nature 406, 151-157. doi: 10.1038/35018003

Tans-Kersten, J., Huang, H. Y., and Allen, C. (2001). Ralstonia solanacearum needs motility for invasive virulence on tomato. J. Bacteriol. 183, 3597-3605. doi: 10.1128/JB.183.12.3597-3605.2001

Tseng, Y. H., Lo, M. C., Lin, K. C., Pan, C. C., and Chang, R. Y. (1990). Characterization of filamentous bacteriophage $\phi \mathrm{Lf}$ from Xanthomonas campestris pv. campestris. J. Gen. Virol. 71, 1881-1884. doi: 10.1099/0022-1317-71-81881

Vasse, J., Genin, S., Frey, P., Boucher, C., and Brito, B. (2000). The hrpB and hrpG regulatory genes of Ralstonia solanacearum are required for different stages of the tomato root infection process. Mol. Plant Microbe Interact. 13, 259-267. doi: 10.1094/MPMI.2000.13.3.259

Wang, Y. A., Yu, X., Overman, S., Tsuboi, M., Tomas, G. J., Egelman, E. H. (2006) The structure of a filamentous bacteriophage. J. Mol. Biol. 361, 209-215. doi: 10.1016/j.jmb.2006.06.027

Webb, J. S., Lau, M., and Kjelleberg, S. (2004). Bacteriophage and phenotypic variation in Pseudomonas aeruginosa biofilm development. J. Bacteriol. 186, 80668073. doi: 10.1128/JB.186.23.8066-8073.2004
Xue, H., Xu, Y., Boucher, Y., and Polz, M. F. (2012). High frequency of a novel filamentous phage, VCY $\phi$ within an environmental Vibrio cholerae population. Appl. Environ. Microbiol. 78, 28-33. doi: 10.1128/AEM.06297-11

Yamada, T. (2011). "Bacteriophages of Ralstonia solanacearum: their diversity and utilization as biocontrol agents in agriculture," in Bacteriophages, ed. I. Kurtboke (Rijeka, Croatia: In Tech-Open Access Publisher), 113-139.

Yamada, T., Kawasaki, T., Nagata, S., Fujiwara, A., Usami, S., and Fujie, M. (2007). New bacteriophages that infect the phytopathogen Ralstonia solanacearum. Microbiology 153, 2630-2639. doi: 10.1099/mic.0.2006/00 1453-0

Yao, J., and Allen, C. (2007). The plant pathogen Ralstonia solanacearum needs aerotaxis for normal biofilm formation and interactions with its tomato host. J. Bacteriol. 189, 6415-6424. doi: 10.1128/JB.00398-07

Conflict of Interest Statement: The author declares that the research was conducted in the absence of any commercial or financial relationships that could be construed as a potential conflict of interest.

Received: 15 August 2013; accepted: 14 October 2013; published online: 04 November 2013.

Citation: Yamada T (2013) Filamentous phages of Ralstonia solanacearum: doubleedged swords for pathogenic bacteria. Front. Microbiol. 4:325. doi: 10.3389/fmicb. 2013.00325

This article was submitted to Evolutionary and Genomic Microbiology, a section of the journal Frontiers in Microbiology.

Copyright (c) 2013 Yamada. This is an open-access article distributed under the terms of the Creative Commons Attribution License (CC BY). The use, distribution or reproduction in other forums is permitted, provided the original author(s) or licensor are credited and that the original publication in this journal is cited, in accordance with accepted academic practice. No use, distribution or reproduction is permitted which does not comply with these terms. 